\title{
REVISED MicroRNA levels quantified in whole blood varies from
}

\section{PBMCs [version 3; peer review: 2 approved, 1 not approved]}

\author{
Sadaf Atarod, Hannah Smith, Anne Dickinson, Xiao-Nong Wang
}

Haematological Sciences, Institute of Cellular Medicine, Newcastle University, Newcastle upon Tyne, NE2 4HH, UK

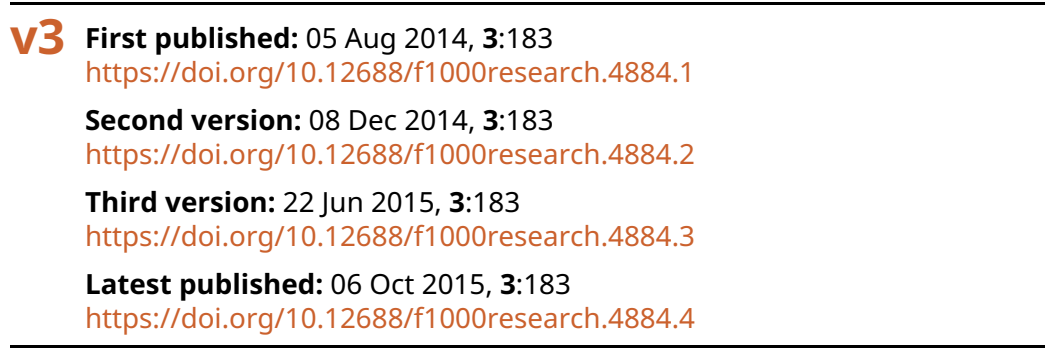

\section{Abstract}

MicroRNAs are non-coding RNAs that negatively regulate mRNA expression and play significant roles in both health and disease. Differential microRNA expression has been used to aid diagnosis and discriminate disease stages. The accuracy and reliability of microRNA expression measurement is of utmost importance. Quantification of microRNA expression in human peripheral blood is commonly detected using total RNA extracted via different methods. To date, no convincing data are available showing whether microRNA quantification results can be influenced by the use of total RNA extracted from whole blood or peripheral blood mononuclear cells (PBMCs). This study examined miR-146a-5p and miR-155-5p expression using total RNA extracted in parallel from whole blood and PBMCs of 14 healthy volunteers. The data showed that the quantification of miRNA using total RNA extracted from whole blood varied from that of PBMCs, indicating that the miRNA expression was a result of all the different cell-types present in whole blood. Our results suggested that the source of total RNA and the statistical analyses performed are crucial considerations when designing miRNA research.

Keywords

MicroRNAs, PBMC

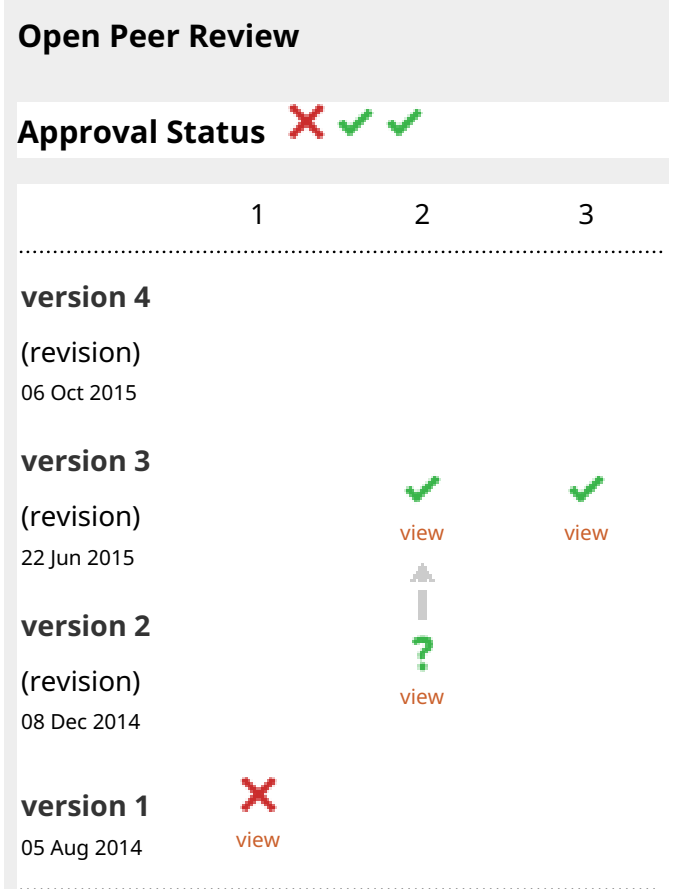

\footnotetext{
1. Kenneth Whitaker Witwer ID, Johns

Hopkins University, Baltimore, USA

2. Steven O'Reilly, Durham University, Durham, UK

3. Stefano Casola, Institute of Molecular Oncology Foundation (IFOM Institute), Milan, Italy
}

Any reports and responses or comments on the article can be found at the end of the article. 
Corresponding author: Sadaf Atarod (s.atarod@newcastle.ac.uk)

Competing interests: No competing interests were disclosed.

Grant information: This work was supported by the Newcastle University and the FP7 Marie Curie Initial Training Network CELLEurope (Contract No: 315963).

The funders had no role in study design, data collection and analysis, decision to publish, or preparation of the manuscript.

Copyright: ( 2015 Atarod S et al. This is an open access article distributed under the terms of the Creative Commons Attribution License, which permits unrestricted use, distribution, and reproduction in any medium, provided the original work is properly cited. Data associated with the article are available under the terms of the Creative Commons Zero "No rights reserved" data waiver (CC0 1.0 Public domain dedication).

How to cite this article: Atarod S, Smith $\mathrm{H}$, Dickinson $\mathrm{A}$ and Wang XN. MicroRNA levels quantified in whole blood varies from PBMCs [version 3; peer review: 2 approved, 1 not approved] F1000Research 2015, 3:183 https://doi.org/10.12688/f1000research.4884.3

First published: 05 Aug 2014, 3:183 https://doi.org/10.12688/f1000research.4884.1 


\section{REVISED Amendments from Version 2}

This version has a Supplementary Figure included in it to confirm that there was no contamination in the no-template controls.

See referee reports

\section{Introduction}

MicroRNAs (miRNAs) are cell- and therefore tissue-specific, and their expression levels impact protein translation (Sood et al., 2006). Nearly, 2000 microRNA (miRNA) sequences have been identified in humans (Kozomara \& Griffiths-Jones, 2014). Numerous studies have reported specific miRNA expression levels in peripheral blood (PB) as markers of disease (Mookherjee \& El-Gabalawy, 2013; Patnaik et al., 2012). Although miRNA expression levels could be inevitably influenced by the way that total RNA is extracted, often studies reporting differential miRNA expression levels fail to emphasise the impact of RNA extraction methods. This could at least partially lead to significant controversies and inconsistencies in the literature related to miRNA research.

PAXgene Blood RNA System (PAXgene Blood RNA Tube and PAXgene Blood miRNA Kit [PAXM]) has been the gold standard for $\mathrm{PB}$ collection as the stabilising reagent present in the tube prevents RNA degradation and inhibits changes in gene expression due to the collection procedure (Viprey et al., 2012). However, it should be emphasised that the miRNA expression detected in whole blood is the overall outcome from total hematocytes rather than the lymphocyte fraction only. Recent studies have shown that erythrocytes also contain a high proportion of miRNAs (Hamilton, 2010). Bayatti et al. have shown that the presence of globin (globular proteins such as haemoglobin) in PB can impact RNA expression and that globin depletion can decrease total RNA quality and yield in particular when extraction is performed using the PAXgene Blood RNA kit (Bayatti et al., 2014). However, it is necessary to evaluate the miRNA expression levels in an independent cohort, as miRNAs are in general highly stable, and therefore their expression might not be affected by globin treatment. A recent research has reported that miRNA expression levels detected using whole blood correlated to that of PBMCs when using PAXgene Blood RNA System and mirVana miRNA kit (MM), respectively (Mookherjee \& El-Gabalawy, 2013). The study did not show that the expression levels are comparable or that they agree with each other using the recommended Bland-Altman method comparison statistical test (Bland \& Altman, 1986). This has raised some confusion as the PAXgene Blood RNA system extracts from whole blood while MM from PBMCs. Performing a comprehensive statistical analysis is required when two methods are compared (Burd, 2010).

In this investigation we have measured the expression of miR-146a-5p and miR-155-5p which have extensively been investigated in whole blood and PBMCs due to their critical functions in the innate and adaptive immune system (Curtale et al., 2010; Schulte et al., 2013). We sought to compare miRNA expression levels in peripheral blood collected from 14 healthy volunteers using both extraction methods (PAXM and mirVana PARIS [MP]). We sought to address (i) whether miRNA expression detected in whole blood is comparable to that of isolated PBMCs and (ii) to detect the presence of haemolysis in whole blood and PBMCs. The experimental design for this investigation is depicted in Figure 1.

\section{Materials and methods}

Volunteer cohort

Whole blood was collected from 14 healthy volunteers following approval from the Newcastle and North Tyneside 2 Research Ethics Committee (STEMDIAGNOSTICS: REC-07/H0906/131) and informed consent was obtained from every volunteer for both blood collection and miRNA testing. Samples from 14 healthy volunteers (5 males and 9 females) were used to quantify miRNA expression in whole blood and PBMC.

\section{Sample collection and storage}

PB (2.5 ml) was collected in PAXgene Blood RNA Tubes (PreAnalytiX GmbH, Switzerland [Catalog No: 762165]) containing an RNA stabilising agent that lysed the blood cells and stabilised the intracellular RNA. The tubes were stored at $-20^{\circ} \mathrm{C}$ until processed. PB was also collected in sodium (Na)-heparin (Sigma, UK) containing tubes for peripheral blood mononuclear cell isolation using graduated centrifugation over Lymphoprep ${ }^{\mathrm{TM}}$ (STEMCELL Technologies, Manchester, UK). The cells were then stored at $-4^{\circ} \mathrm{C}$ before extraction. Isolated PBMCs were cryopreserved by re-suspension in freezing solution containing $350 \mathrm{ml}$ of RPMI 1640 (Sigma-Aldrich, UK), $20 \%$ fetal calf serum and $10 \%$ dimethyl sulfoxide (NBS Biologicals, UK) and stored in Cryovials at $-80^{\circ} \mathrm{C}$.

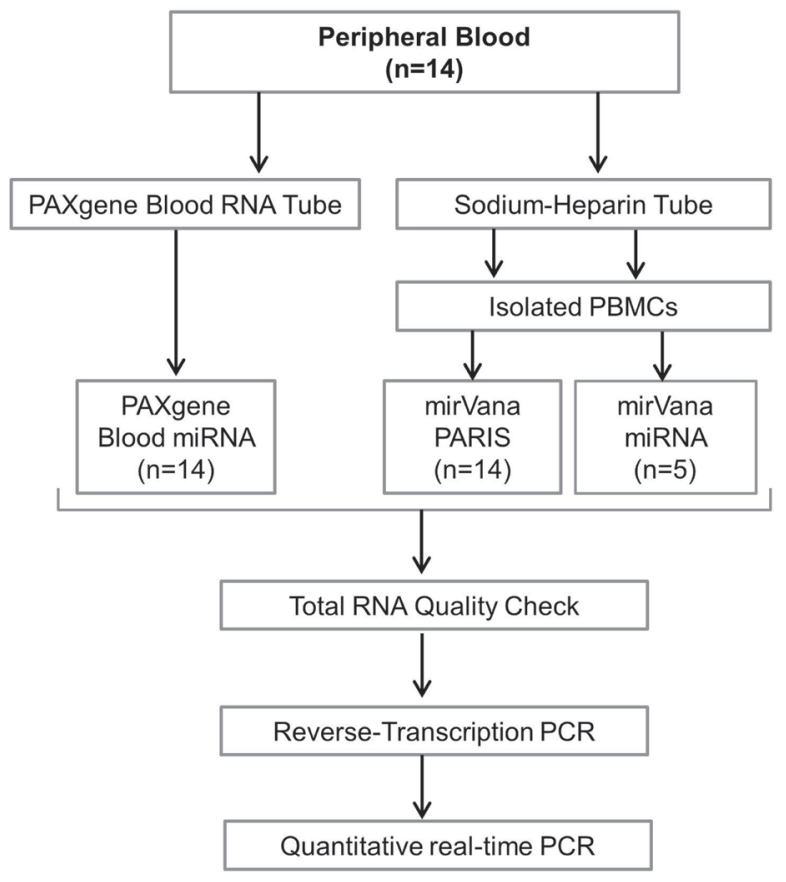

Figure 1. Experimental design. Peripheral blood was collected into various tubes as illustrated. Total RNA was extracted from whole blood using the PAXgene blood miRNA kit and from PBMCs using the mirVana Paris and mirVana isolation kits, respectively. The total RNA contained the miRNA population which was measured by firstly performing reverse transcription and then quantitative real-time PCR. 
Total RNA extraction from peripheral blood

Total RNA was extracted from isolated PBMCs using the (i) $m i r V a n a^{\mathrm{TM}}$ PARIS ${ }^{\mathrm{TM}}$ kit (MP) and (ii) mirVana ${ }^{\mathrm{TM}}$ miRNA Isolation kit (MM) (Ambion, USA [Catalog Nos: AM1556 and AM1560, respectively]) according to the manufacturer's protocol. PAXgene Blood RNA Tubes were incubated overnight at room temperature to increase the RNA yield. Total RNA extraction from whole blood was performed using the PAXgene Blood miRNA kit (PAXM) (PreAnalytiX GmBh, Switzerland [Catalog No: 763134]), according to the manufacturer's protocol. Aseptic techniques were followed at all stages of extraction. Total RNA quality and concentration were assessed using NanoDrop ND-1000 spectrophotometer (Thermo Fischer Scientific, MA). The absorbance ratios $260 \mathrm{~nm} / 280 \mathrm{~nm}$ and $260 \mathrm{~nm} / 230 \mathrm{~nm}$ were analysed to determine the purity of total RNA.

Reverse transcription and quantitative real-time PCR MicroRNA specific cDNA was synthesised from 10 ng total RNA extracted from isolated PBMCs and whole blood using TaqMan MicroRNA reverse transcription (RT) kit (Applied Biosystems, Life Technologies, USA [Catalog No: 4366596]) as per the manufacturer's protocol. The same RNA concentration was used for all the reactions. Hydrolysis probes were used for cDNA synthesis (Assay IDs: miR-155-5p-5p: 000479, miR-146a-5p: 000468, miR451-5p: 001141, miR-23a-3p: 000399, SNORD49A [RNU49]: 001005, SNRNP27 [U6]: 001973, SNORA74A [RNU19]: 001003 and SNORD48 [RNU48]: 001006). No-enzyme control (NEC) and a negative template control (NTC) were run for every extraction and $\mathrm{RT}$ reaction set. The samples were then run on a thermal cycler at four different holding temperatures: $16^{\circ} \mathrm{C}$ for 30 minutes, $42^{\circ} \mathrm{C}$ for 30 minutes, $85^{\circ} \mathrm{C}$ for 5 minutes and finally at $4^{\circ} \mathrm{C}$ until storage at $-4^{\circ} \mathrm{C}$.

Quantitative real-time PCR (qPCR) was performed using the TaqMan method and hydrolysis probes mentioned above (Applied Biosystems by Life Technologies, CA, USA) according to the manufacturer's protocol. Each sample was run in triplicate and every plate contained the NTC from the RT step and the qPCR step as well as NEC on a 7900HT Fast Real-Time PCR System (Life Technologues, CA, USA).

\section{Data analysis}

The qPCR results were analysed using SDS v2.4 software and normalised using SNORD48 as the reference control which was selected by testing a panel of four controls for stable expression within the whole blood and PBMCs. The comparative $\Delta \Delta \mathrm{C}_{\mathrm{q}}$ method was used to calculate fold-changes $\left(\Delta \mathrm{C}_{\mathrm{q}}=\mathrm{C}_{\mathrm{q} \text { microRNA of interest }}-\mathrm{C}_{\mathrm{q} \text { reference control }}\right.$ ' Relative Quantification [RQ] $=2^{-\Delta C \mathrm{Cq}}$ and LOG transformed = $\mathrm{LOG}_{2} \mathrm{RQ}$ ). Fold-change was logarithm-transformed as qPCR data are non-linear (exponential), and is transformed to decrease the heterogeneity of variance (McDonald, 2009) and also to identify the outliers present in the data (Rieu \& Powers, 2009). Standard curves for three samples from both PAXM and MP were generated and a $95 \%$ confidence interval slope of the line was used to calculate the PCR efficiency (E) using the standard formula; $\mathrm{E}=10^{-1 / \text { Slope }}$ and $\%$ efficiency $=(E-1) \times 100$. Mean efficiency was then calculated. Results were analysed and plotted using GraphPad PRISM v5.0 software (GraphPad Software, Inc, USA). Mann-Whitney $\mathrm{U}$ t-test was used to assess difference between two groups and KruskalWallis one-way analysis of variance (ANOVA) for multiple groups. Spearman's test was used to determine correlation. Bland-Altman was performed to test whether two methods agreed and if one could be interchanged with another. Significance was set at $p<0.05$.

\section{Results}

Quality of RNA extracts

Total RNA was extracted using three different extraction methods; PAXM (PAXgene Blood miRNA), MP (mirVana PARIS) and MM (miRVana miRNA) from whole blood and PBMCs. RNA purity was assessed by detecting the absorbance ratios at $260 \mathrm{~nm} / 280 \mathrm{~nm}$ and at $260 \mathrm{~nm} / 230 \mathrm{~nm}$. The ratio $(260 \mathrm{~nm} / 280 \mathrm{~nm})$ for whole blood was $1.95-2.35$ and for isolated PBMCs was $2.00-2.27$ (both MP and $\mathrm{MM}$ respectively). The ratios were $\geq 1.8-2.0$; thus extraction was free from protein contamination which is usually absorbed at $280 \mathrm{~nm}$. Absorbance ratios detected at $260 \mathrm{~nm} / 230 \mathrm{~nm}$ showed ratios below the accepted contamination-free range of $1.5-2.0$ (PBMCs: $0.18-1.83$ and whole blood: $0.15-1.49$ ). Therefore, the samples may have been affected by contaminants absorbed at $230 \mathrm{~nm}$ such as guanidine isothiocyanate present in all the three extraction kits. For RNA purity, the peak of each total RNA plot was also analysed as it could indicate contamination by phenol and/ guanidine isothiocyanate. PBMCs had plot peaks at $260 \mathrm{~nm}$ thus confirming contaminant-free samples. However, peaks at $260 \mathrm{~nm}$ were absent for total RNA extracted using the PAXM (Figure 2). This further suggests that guanidine salts may have been the cause of contamination in whole blood samples extracted using PAXM.
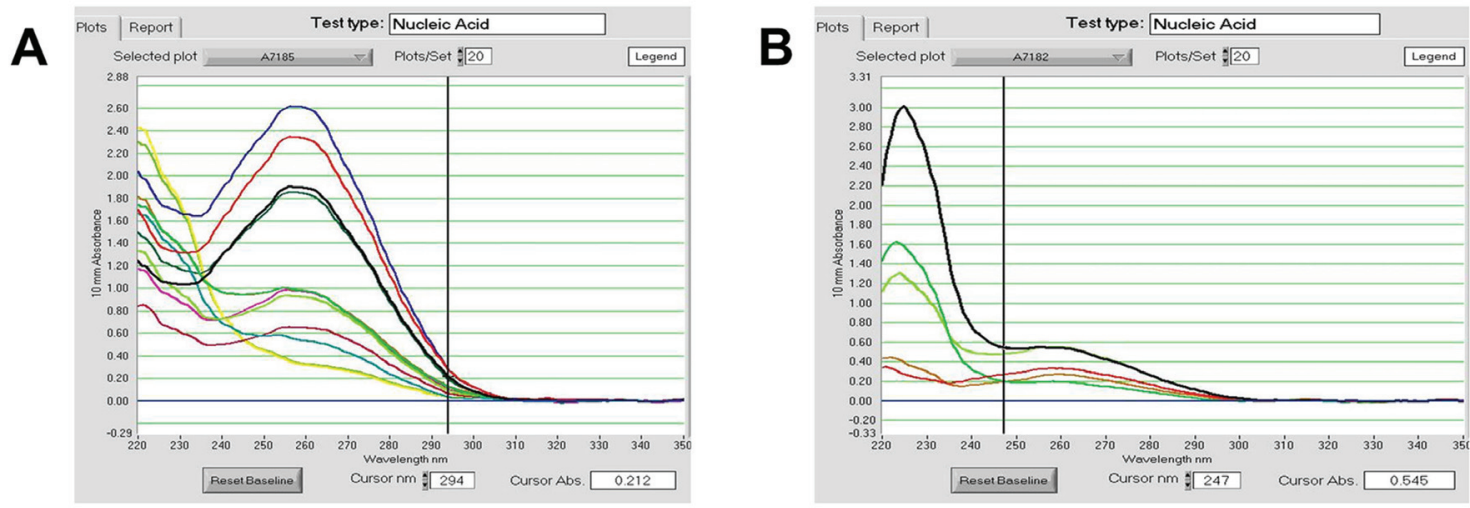

Figure 2. Representative graphical plots of total RNA extracted. (A) PAXM: analysis of whole blood showed peaks positioned at $230 \mathrm{~nm}$ $(n=10)$ and (B) MP: analysis of PBMCs showed peaks positioned at $260 \mathrm{~nm}(\mathrm{n}=5)$. 
Quantitative PCR quality controls

NEC and NTC controls were used to ensure that the RT and qPCR reactions were contaminant-free. Each control displayed no amplification $(\mathrm{Cq}>36)$. This was particularly important for total RNA extracted from PBMCs, as they were non-DNase treated. Thus, any amplification in the controls may have suggested either nonspecific binding of primers or presence of contamination such as genomic DNA. In addition we calculated the average efficiency of our real-time qPCR reactions ( $\mathrm{E}=97.8 \%$ ) which confirmed absence of reaction inhibitors such as heparin that was specifically used for the collection of peripheral blood from patients for the downstream PBMC isolation. (See Supplementary Figure 1, that shows there was no contamination in the samples with a $\mathrm{Cq}$ value equal to 40 i.e. no amplification). Determining qPCR efficiency is important as inhibitory compounds can affect miRNA expression and result in false positives.

\section{Reference control for microRNA expression in peripheral} blood

We determined the most appropriate reference gene for this investigation by testing a panel of four controls that have been known for stable expression (SNORD49A [RNU49], SNRNP27 [U6], SNORA74A [RNU19] and SNORD48 [RNU48]) (Dataset a). Our results showed that SNORD48 expression was the most stable in total RNA extracted via both the PAXM (Figure 3A) and MP (Figure 3B) method. Therefore, SNORD48 was used as the reference control to normalise miR-146a-5p and miR-155-5p expression in each sample.

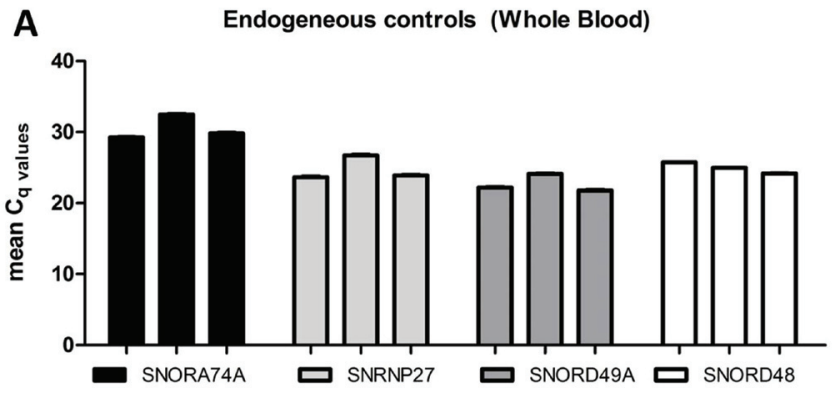

B

Endogeneous controls (PBMC)

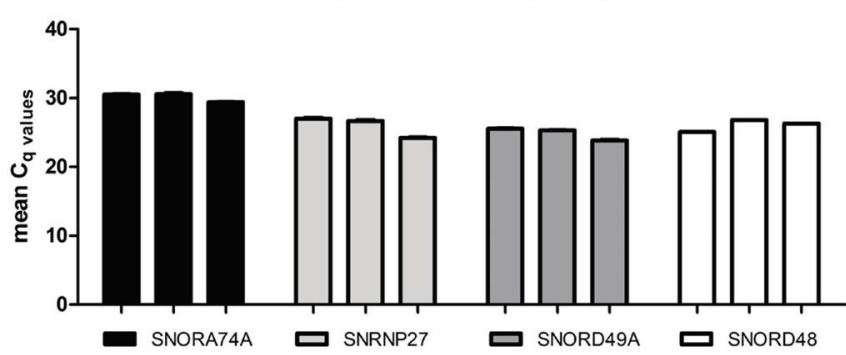

Figure 3. Expression of endogenous controls in total RNA extracted. A panel of four stably expressed miRNAs were selected and quantified to identify the most stable control for normalisation of miR-146a-5p and miR-155-5p in paired samples $(n=3)(\mathbf{A})$ whole blood and (B) PBMCs. The standard error of the mean is shown by the error bars for demonstration of technical variability.
Whole blood extracted using PAXM exhibits higher degree of haemolysis

Erythrocyte haemolysis has been reported to alter miRNA measurements in whole blood, plasma, serum and tissues (McDonald et al., 2011; Pritchard et al., 2012). The total RNA extracted using the three different methods was examined for degree of haemolysis by quantifying miR-451-5p and miR-23a-3p (Dataset b). Normalised $\Delta \mathrm{Cq}$ values (miR-23a-3p - miR-451-5p) greater than seven were considered as an indicator of haemolysis. Our results showed that there was a significantly high degree of haemolysis in the total RNA extracted using the PAXM method with the $\triangle \mathrm{Cq}$ values in the range of 9 - 11. Haemolysis was low in total RNA extracted using either of MP or MM, $\Delta \mathrm{Cq}>3$ (Figure 4).

\section{MicroRNA expression in whole blood and PBMCs}

With inclusion of stringent quality controls, we assessed miR-146a-5p and miR-155-5p expression in total RNA extracted in parallel from whole blood using PAXM $(\mathrm{n}=14)$ and PBMCs using MP $(\mathrm{n}=14)$ (Dataset c). Our results showed that there was no correlation (Figure 5, miR-146a-5p: r=-0.352, $\mathrm{p}=0.217$ and miR-155-5p: $\mathrm{r}=0.380, \mathrm{p}=0.180$ ) between PAXM and MP in the expression of both miR-146a-5p and miR-155-5p. In a PCR reaction, it is assumed that the target expression doubles at every reaction cycle. Bland-Altman analysis also showed that the two methods did not agree as the bias was greater than 1 which equated to more than one qPCR cycle difference between the two methods (Figure 5A and 5B). Mookherjee et al. had used MM to extract total RNA from PBMCs, then correlated miRNA expression between the PAXM and MM method

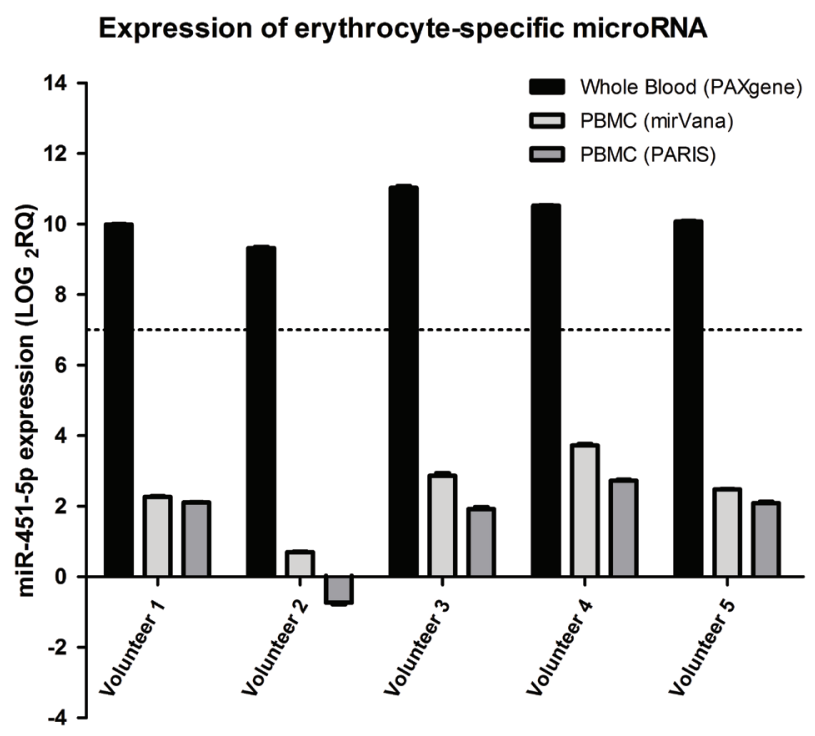

Figure 4. Degree of haemoloysis in total RNA extracted via the three extraction methods. Total RNA extracted using the PAXgene miRNA isolation kit. $A$ threshold of $L O G_{2} R Q$ greater than 7 was indicative of haemolysis (dashed line). The degree of haemolysis was calculated by measuring the difference between miR-451-5p and miR-23a-3p expression. All data $(n=5)$ have been log-transformed. The standard error of the mean is shown by the error bars for demonstration of technical variability. 
(Mookherjee \& El-Gabalawy, 2013). To eliminate the possibility that using MP was the reason for the non-correlation and disagreement, we tested the three different extraction methods (PAXM, MP and $\mathrm{MM}$ ) for both miRNAs in a randomly selected cohort of five healthy volunteers (Figure 6) (Dataset d). The results demonstrated that PAXM and MP as well as PAXM and MM did not correlate nor agreed with one another. However, MP and MM methods agreed with each other and could therefore be interchanged as the bias between the two methods for both miR-146a-5p and miR-155-5p was only $0.769(\mathrm{SD}=0.307)$ and $0.892(\mathrm{SD}=0.802)$, respectively. Interestingly, normalised miRNA expression was significantly different only between PAXM and MM methods (miR-146a-5p and miR-155-5p: $\mathrm{p}<0.01)$. There was higher miRNA expression in PBMCs than in whole blood for both miRNAs (Figure 7).
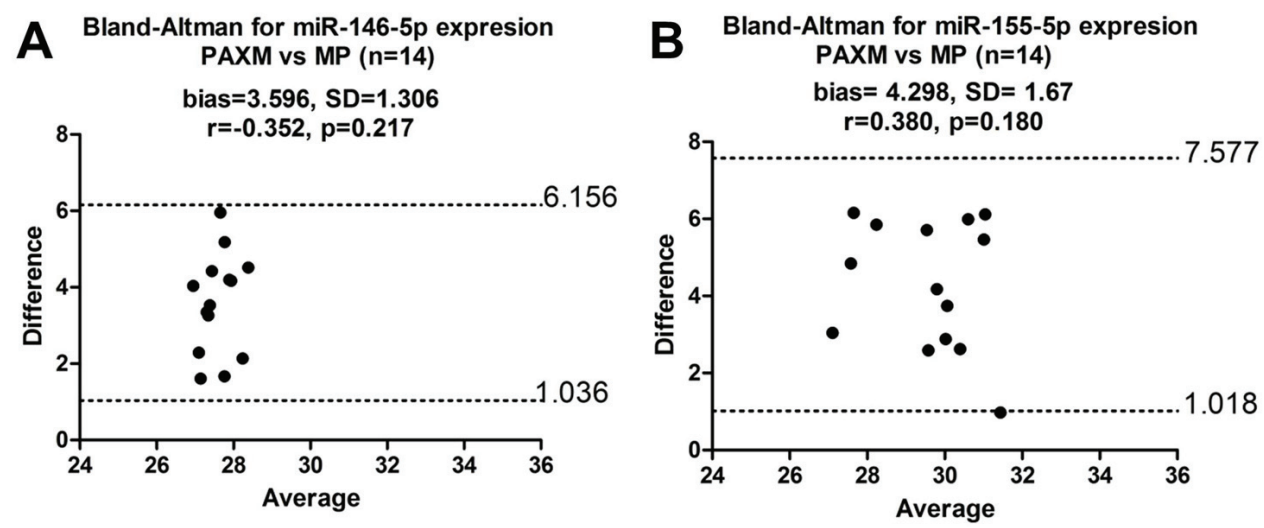

Figure 5. Bland-Altman plots for miRNA expression from whole blood and PBMCs. Total RNA was extracted ( $\mathrm{n}=14)$ using PAXM and MP for (A) miR-146a-5p and (B) miR-155-5p expression. MicroRNA expression is within the limits of agreement but the bias is greater than one showing high disagreement between PAXM and MP. $r$ indicates Spearman correlation. SD: Standard Deviation and bias is the mean difference. $C_{q}$ values were used for this analysis. Dashed lines show the 95\% lower and upper limits of agreement.
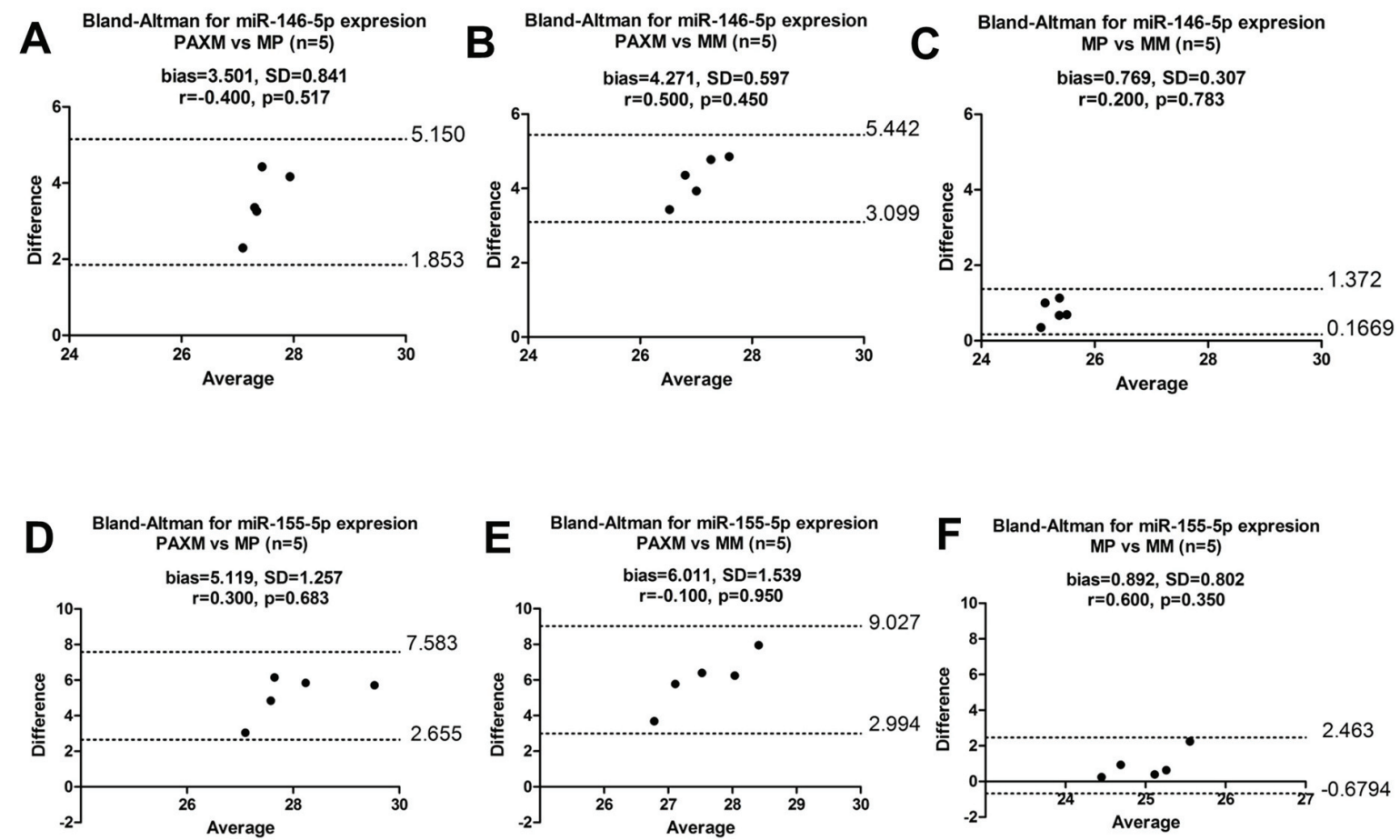

Figure 6. Bland-Altman plots for miR-146a-5p and miR-155-5p expression in whole blood and PBMCs. The three methods were all compared for miR-146a-5p as (A) PAXM vs MP (B) PAXM vs MM and (C) MP vs MM as well as miR-155-5p (D) PAXM vs MP (E) PAXM vs MM (F) MP vs MM MicroRNA expression is within the limits of agreement but the bias is greater than one showing high disagreement between PAXM and MP. Bias is lower than one for MP and MM, thus the two methods agree with one another. $r$ indicates Spearman correlation. SD: Standard Deviation and bias is the mean difference. $C_{q}$ values were used for this analysis. Dashed lines show the $95 \%$ lower and upper limits of agreement. 

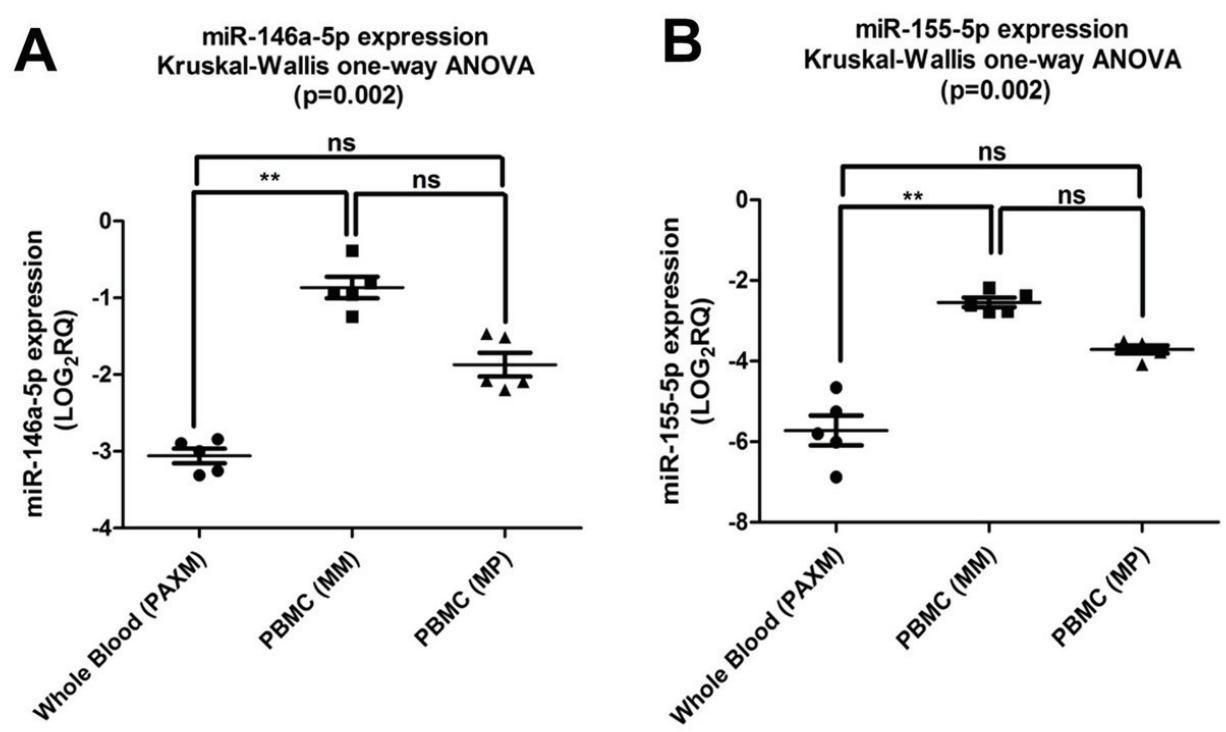

Figure 7. Normalised miRNA expression comparison from whole blood and PBMCs. (A) miR-146a-5p and (B) miR-155-5p expression. MicroRNA expression is significantly varied across all the three different groups ( $p=0.002)$. MicroRNA expression is higher in PBMCs extracted via either MP or MM method in comparison to whole blood. ${ }^{* *} \mathrm{p}<0.01$ and ns: not significant.

\section{Dataset 1. Data of miRNA extraction methods from whole blood and PBMCs}

http://dx.doi.org/10.5256/f1000research.4884.d33496

Dataset (a) shows the expression of all endogenous controls used in the study. Dataset (b) shows the degree of cell hemolysis using different extraction kits. Datasets (c)-(d)-(e) contain data of miR-146a-5p and miR-155-5p expression in whole blood and PBMCs in different samples. Complete dataset legends can be found in the text file.

\section{Discussion}

MicroRNA expression levels are used to classify diseases and also to distinguish the diseased from the healthy population. However, lack of uniform detection protocols has led to controversies and inconsistencies in miRNA research. There is also lack of recognition for the presence of miRNAs from erythrocytes and other celltypes when using whole blood for total RNA extraction processes and downstream miRNA studies. In most investigations, PBMCs are considered as the major cellular sources for miRNAs. This work was conducted to elucidate the difference between total RNA extracted from whole blood and PBMCs for miRNA expression level studies and also to highlight the importance of protocol standardization.

RT-qPCR was performed to examine whether the expression of miR-146a-5p and miR-155-5p in whole blood and PBMC agreed with one another. Our results showed that there was no agreement between PAXM and both MP and MM for miR-146a-5p and miR155-5p expression. PBMCs constitute only a fraction of the cells present in PB and therefore lack granulocytes, platelets and erythrocytes (Min et al., 2010). Due to the unique miRNA expression pattern in each cell-type the relative proportions of cells in blood may have an effect on the overall miRNA expression profile and the expression of their protein targets (Min et al., 2010). Some studies have shown that mature miRNA expression signature in erythrocytes is similar to that in whole blood while different when compared to PBMCs (Chen et al., 2008). MiR-451-5p is a marker of erythrocytes (Rasmussen et al., 2010) and miR-23a-3p is unaffected by haemolysis (Blondal et al., 2013). We have shown that there is lower miR-146a-5p and miR-155-5p expression in whole blood compared to PBMCs demonstrating that the total RNA extracted from PBMCs does not reflect that detected in whole blood. Several studies have shown that total RNA yield from whole blood decreases after the use of DNase step in the PAXM protocol (Asare et al., 2008; Bayatti et al., 2014; Debey et al., 2004). However, if PAXM total RNA is not DNase-treated, there may be a possibility of DNA contamination. Contrary to our results, Mookherjee et al. found a linear correlation between miR146a-5p and miR-155-5p expression in whole blood and isolated PBMCs collected from a healthy population (Mookherjee \& El-Gabalawy, 2013). In their work, they did not measure the degree of haemolysis in the samples, which may partly explain the discrepancy between the two studies. Furthermore, our study compared both the strength (correlation) and level of agreement between the two methods whilst Mookherjee et al. examined only the correlation (Bland \& Altman, 1986). This highlights the importance of performing the correct statistics when two methods are compared with regards to their equivalence and interchangeability (Burd, 2010).

In clinical practice, it is easier to collect PB in PAXgene Blood RNA tubes as they have a shelf-life of two to five years without any RNA degradation. Immediate stabilisation is vital as storage of blood cells induce changes in the miRNA composition 
(Gaarz et al., 2010). Thus, extraction methods from whole blood must be optimised to either eliminate erythrocyte contamination or consider the expression as cumulative and design downstream experiments for miRNA protein target studies accordingly.

In conclusion, our study showed differences in miR-146a-5p and miR-155-5p expression in isolated PBMCs and whole blood. We suggest that PBMCs are not the ideal source to study and correlate miRNA protein targets where the miRNA expression had been measured in whole blood as the miRNA expression pattern in whole blood is not comparable to that in PBMCs. We also highlight the importance of having a stringent set of technical controls and performing the correct statistics to increase the reliability and reproducibility of miRNA expression studies.

\section{Consent}

All participants to the study provided informed written consent for molecular testing and publication of the data.

\section{Data availability}

F1000Research: Dataset 1. Data of miRNA extraction methods from whole blood and PBMCs, 10.5256/f1000research.4884. d33496 (Atarod et al., 2014).

\section{Author contributions}

S.A performed the experiments, analysed the results and wrote the manuscript. H.S. performed part of the experiments. A.M.D provided constructive comments for the discussion. XN.W interpreted the results and wrote the manuscript. All authors revised the manuscript and agreed to the final content.

\section{Competing interests}

No competing interests were disclosed.

\section{Grant information}

This work was supported by the Newcastle University and the FP7 Marie Curie Initial Training Network CELLEurope (Contract No: 315963).

The funders had no role in study design, data collection and analysis, decision to publish, or preparation of the manuscript.

\section{Acknowledgements}

We would like to thank all the volunteers who donated blood for this study. We would also like to thank Dr Clare Lendrem and Dr Kim Pearce for their statistical advice.

\section{Supplementary Materials}

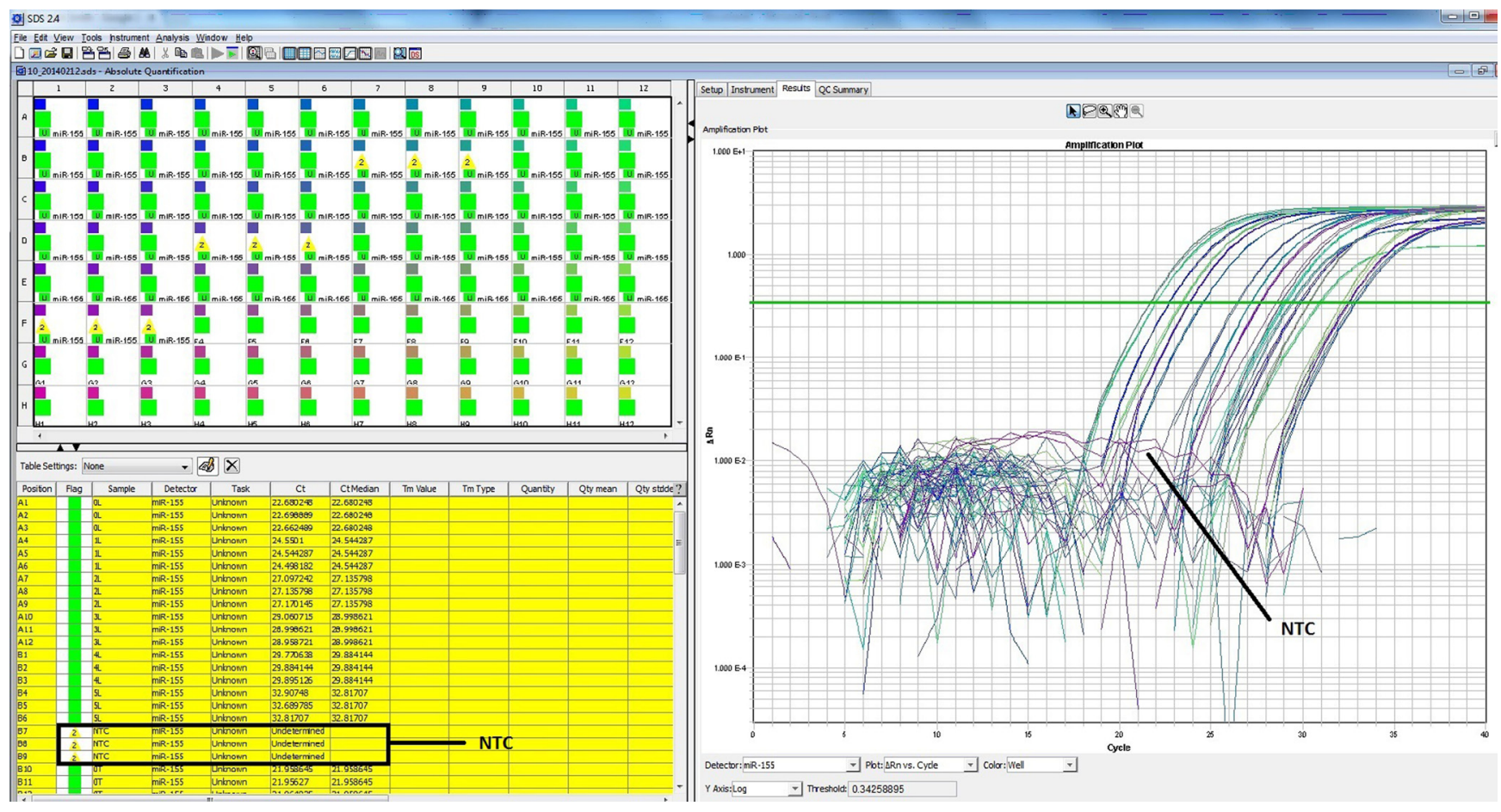

Supplementary Figure 1. RT-qPCR amplification curves. There was no amplification observed in the no-template control (NTC) sample. 
Asare AL, Kolchinsky SA, Gao Z, et al.: Differential gene expression profiles are dependent upon method of peripheral blood collection and RNA isolation. BMC Genomics. 2008; 9: 474.

PubMed Abstract | Publisher Full Text | Free Full Text

Atarod S, Smith H, Dickinson A, et al.: Data of miRNA extraction methods from whole blood and PBMCs. F1000Research. 2014.

Data Source

Bayatti N, Cooper-Knock J, Bury JJ, et al:: Comparison of blood RNA extraction methods used for gene expression profiling in amyotrophic lateral sclerosis. PLOS One. 2014; 9(1): e87508.

PubMed Abstract | Publisher Full Text | Free Full Text

Bland JM, Altman DG: Statistical methods for assessing agreement between two methods of clinical measurement. Lancet. 1986; 1(8476): 307-310.

PubMed Abstract | Publisher Full Text

Blondal T, Jensby Nielsen S, Baker A, et al.: Assessing sample and miRNA profile quality in serum and plasma or other biofluids. Methods (San Diego, Calif). 2013; 59(1): S1-6.

PubMed Abstract | Publisher Full Text

Burd EM: Validation of laboratory-developed molecular assays for infectious diseases. Clin Microbiol Rev. 2010; 23(3): 550-576. PubMed Abstract | Publisher Full Text | Free Full Tex

Chen SY, Wang Y, Telen MJ, et al.: The genomic analysis of erythrocyte microRNA expression in sickle cell diseases. PLoS One. 2008; 3(6): e2360. PubMled Abstract | Publisher Full Text | Free Full Text

Curtale G, Citarella F, Carissimi C, et al.: An emerging player in the adaptive immune response: microRNA-146a is a modulator of IL-2 expression and activation-induced cell death in T lymphocytes. Blood. 2010; 115(2): 265-273. PubMed Abstract | Publisher Full Text

Debey S, Schoenbeck U, Hellmich M, et al.: Comparison of different isolation techniques prior gene expression profiling of blood derived cells: impact on physiological responses, on overall expression and the role of different cell types. Pharmacogenomics J. 2004; 4(3): 193-207.

PubMed Abstract | Publisher Full Text

Gaarz A, Debey-Pascher S, Classen S, et al: Bead array-based microRNA expression profiling of peripheral blood and the impact of different RNA isolation approaches. $J$ Mol Diagn. 2010; 12(3): 335-344.

PubMed Abstract | Publisher Full Text | Free Full Text

Hamilton AJ: MicroRNA in erythrocytes. Biochem Soc Trans. 2010; 38(Pt 1): 229-231. PubMed Abstract | Publisher Full Text

Ji J, Shi J, Budhu A, et al.: MicroRNA expression, survival, and response to interferon in liver cancer. N Engl J Med. 2009; 361(15): 1437-1447.

PubMed Abstract | Publisher Full Text | Free Full Tex
Kozomara A, Griffiths-Jones S: miRBase: annotating high confidence microRNAs using deep sequencing data. Nucleic Acids Res. 2014; 42(Database issue): D68-73.

PubMed Abstract | Publisher Full Text | Free Full Text

McDonald JH: Handbook of Biological Statistics. Sparky House Publishing, Baltimore, Maryland. 2009.

Reference Source

McDonald JS, Milosevic D, Reddi HV, et al: Analysis of circulating microRNA:

preanalytical and analytical challenges. Clin Chem. 2011; 57(6): 833-840. PubMed Abstract | Publisher Full Text

Min JL, Barrett A, Watts T, et al:: Variability of gene expression profiles in human blood and lymphoblastoid cell lines. BMC Genomics. 2010; 11: 96. PubMed Abstract | Publisher Full Text | Free Full Tex

Mookherjee N, El-Gabalawy HS: High degree of correlation between whol blood and PBMC expression levels of miR-155 and miR-146a in healthy controls and rheumatoid arthritis patients. $J$ Immunol Methods. 2013; 400-401: 106-110.

PubMed Abstract | Publisher Full Text

Patnaik SK, Yendamuri S, Kannisto E, et al.: MicroRNA expression profiles of whole blood in lung adenocarcinoma. PLoS One. 2012; 7(9): e46045.

PubMed Abstract | Publisher Full Text | Free Full Text

Pritchard CC, Kroh E, Wood B, et al:: Blood cell origin of circulating microRNAs: a cautionary note for cancer biomarker studies. Cancer Prev Res (Phila). 2012; 5(3): 492-497.

PubMed Abstract | Publisher Full Text | Free Full Text

Rasmussen KD, Simmini S, Abreu-Goodger C, et al:: The miR-144/451 locus is required for erythroid homeostasis. J Exp Med. 2010; 207(7): 1351-1358. PubMed Abstract | Publisher Full Text | Free Full Text

Rieu I, Powers SJ: Real-time quantitative RT-PCR: design, calculations, and statistics. Plant Cell. 2009; 21(4): 1031-1033.

PubMed Abstract | Publisher Full Text | Free Full Text

Schulte LN, Westermann AJ, Vogel J: Differential activation and functional specialization of miR-146 and miR-155 in innate immune sensing. Nucleic Acids Res. 2013: 41(1): 542-553.

PubMed Abstract | Publisher Full Text | Free Full Text

Sood P, Krek A, Zavolan M, et al.: Cell-type-specific signatures of microRNAs on target mRNA expression. Proc Natl Acad Sci U S A. 2006; 103(8): 2746-2751. PubMed Abstract | Publisher Full Text | Free Full Text

Viprey VF, Corrias MV, Burchill SA: 'Identification of reference microRNAs and suitability of archived hemopoietic samples for robust microRNA expression profiling'. Anal Biochem. 2012; 421(2): 566-572.

PubMed Abstract | Publisher Full Text 


\section{Open Peer Review}

\section{Current Peer Review Status:}

\section{Version 3}

Reviewer Report 24 September 2015

https://doi.org/10.5256/f1000research.7159.r10479

(C) 2015 Casola S. This is an open access peer review report distributed under the terms of the Creative Commons Attribution License, which permits unrestricted use, distribution, and reproduction in any medium, provided the original work is properly cited.

\section{Stefano Casola}

Genetics and Epigenetics of Lymphocyte Development and Transformation Research Program, Institute of Molecular Oncology Foundation (IFOM Institute), Milan, Italy

In this manuscript, Atarod and colleagues quantify the expression levels of two candidate miRNAs, mir-146-5p and mir-155-5p, starting from either whole blood (WB) or PBMC. Authors employ quantitative real-time PCR to measure candidate miRNA transcripts from the two biological sources. Different procedures were used to extract total RNA from the two sources, which could, at least in part, affect downstream expression analyses. The study is sound and the data are convincing and relevant for appropriate selection of the blood source from which to measure levels of miRNAs of interest. The conclusions reached by the authors indicate poor correspondence in the expression of mir-146-5p and mir-155-5p when comparing WB to PBMC as biological source. The results by Atarod and colleagues are in apparent contrast with those described in a previous study by Mookherjee \& El-Gabalawy. The reasoning to explain the discrepancy is convincing, and raises important issues related to technical (grade of haemolysis) and methodological aspects (statistical analysis) of the expression analysis that could undermine a correct interpretation of the data.

The title is appropriate and data are presented and discussed in a concise and clear fashion.

The manuscript has benefited from previous rounds of revision, addressing the main concerns raised by the reviewers.

Minor points:

Materials and methods section:

1. Sample collection and storage: Authors state that, after purification, PBMCs were stored at $4^{\circ} \mathrm{C}$. Is that correct? Or, instead it should read $+4^{\circ} \mathrm{C}$ ?

2. Authors state "PBMC were cryopreserved by re-suspension in freezing solution containing $350 \underline{\mathrm{ml}}$ of RPMI 1640.." Authors should replace "350 ml" with (I suspect) 350 $\mathrm{l}$. 
Competing Interests: No competing interests were disclosed.

I confirm that I have read this submission and believe that I have an appropriate level of expertise to confirm that it is of an acceptable scientific standard.

Author Response 25 Sep 2015

Sadaf Atarod, Newcastle University, Newcastle upon Tyne, UK

Thank you for reviewing our manuscript and your valuable comments. Please, find below our replies.

1. We stored the cells on ice, as the extraction was performed soon after the collection. This information has been updated in the revised manuscript.

2. We have updated the revised manuscript with the percentage of each ingredient. We used $70 \%$ RPMI to make up the freezing solution.

Competing Interests: None.

Reviewer Report 23 June 2015

https://doi.org/10.5256/f1000research.7159.r9135

(C) 2015 O'Reilly S. This is an open access peer review report distributed under the terms of the Creative Commons Attribution License, which permits unrestricted use, distribution, and reproduction in any medium, provided the original work is properly cited.

\section{Steven O'Reilly}

School of Biological and Biomedical Sciences, Durham University, Durham, UK

In light of the new version and the author's response to my previous review, I am happy to approve this article.

Competing Interests: No competing interests were disclosed.

I confirm that I have read this submission and believe that I have an appropriate level of expertise to confirm that it is of an acceptable scientific standard.

\section{Version 2}

Reviewer Report 14 May 2015

https://doi.org/10.5256/f1000research.6331.r8664 
(C) 2015 O'Reilly S. This is an open access peer review report distributed under the terms of the Creative Commons Attribution License, which permits unrestricted use, distribution, and reproduction in any medium, provided the original work is properly cited.

\section{Steven O'Reilly}

School of Biological and Biomedical Sciences, Durham University, Durham, UK

This is a very interesting an important study that highlights differences in microRNA expression between whole blood and isolated Peripheral Blood Mononuclear Cells (PBMCs). This is of clear importance where as many authors would be using whole blood microRNA expression and then using isolated PBMCs to draw conclusions on their putative targets. There is clear differences between the two due to the fact of other sources of microRNAs in the whole blood including erythrocytes and platelets and the fact that there are also 'free floating' microRNAs in blood also, likely encased in exosomes. This study is technically sound and I particularly like the fact that the authors calculated the qPCR efficiency. The design is also technically sound.

I would suggest that the authors include a 'screen shot' of the actual amplification curve of the No Template Control (NTC). This will confirm visually that there is no contamination in the samples with a likely CT value $37>$.

Did the authors after lymphoprep isolation notice any difference in expression of the microRNAs before and after cryopreservation? The cryopreservation medium contains DMSO (although low) and we have found this affects certain microRNA expression patterns.

Was there any apparent difference in the levels of the microRNAs correlation with age? I.e. old donors had lower or higher levels of miR-146a because they are immunosenescent (but not diseased)?

Competing Interests: No competing interests were disclosed.

\section{I confirm that I have read this submission and believe that I have an appropriate level of expertise to confirm that it is of an acceptable scientific standard, however I have significant reservations, as outlined above.}

\section{Author Response 12 Jun 2015}

Sadaf Atarod, Newcastle University, Newcastle upon Tyne, UK

Thank you for reviewing our manuscript and your valuable comments. Please, find below our replies.

1. I would suggest that the authors include a 'screen shot' of the actual amplification curve of the No Template Control (NTC). This will confirm visually that there is no contamination in the samples with a likely CT value $37>$.

Thanks for the suggestion. This figure has been added as a supplementary figure to the manuscript. 
2. Did the authors after lymphoprep isolation notice any difference in expression of the microRNAs before and after cryopreservation? The cryopreservation medium contains DMSO (although low) and we have found this affects certain microRNA expression patterns.

We have evaluated the impact of cryopreservation on miRNA expression by extracting total RNA from paired fresh and cryopreserved PBMCs $(n=5)$ and performed RT-qPCR respectively. There was no significant difference between fresh and cryopreserved PBMCs for the expression of miR-146a-5p and miR-155-5p (both $p=0.125$ ). For the figure, please refer to the first version of this paper. In addition, there was no correlation in miRNA expression between fresh and cryopreserved PBMCs with the bias of $<1$ for both miR-146a$5 p$ and miR-155-5p.Thus, in this study, expressions of miR-146a-5p and miR-155-5p in fresh and cryopreserved PBMCs agreed with one another although further studies including more samples and longer period of cryopreservation may provide more informative evidence. Moreover, this finding may not be generalised to other miRNAs as individual miRNAs may have varied susceptibility to cryopreservation.

3. Was there any apparent difference in the levels of the microRNAs correlation with age? I.e. old donors had lower or higher levels of miR-146a because they are immunosenescent (but not diseased)?

In this investigation, there was no correlation between age and miRNA expression levels. However, this investigation cohort included only three older volunteers (age> 55 years). Thus, this finding requires further confirmation in a larger cohort of samples and with older age.

Competing Interests: No competing interest to declare.

\section{Version 1}

Reviewer Report 17 October 2014

https://doi.org/10.5256/f1000research.5214.r6354

(C) 2014 Witwer K. This is an open access peer review report distributed under the terms of the Creative Commons Attribution License, which permits unrestricted use, distribution, and reproduction in any medium, provided the original work is properly cited.

\section{Kenneth Whitaker Witwer}

Molecular and Comparative Pathobiology, Johns Hopkins University, Baltimore, MD, USA

The design of this study is flawed, and several conclusions are not justified by the results. Since the PCR technique seems sound (and the authors are commended for including information such as PCR efficiency), there may be several subcomponents of the study that could be separated out 
and published as a smaller paper (e.g., the results of the gender and freezing experiments).

1. I would encourage the authors to remove immediately the baseless claims about putative problems with the PAXgene reagents or protocol. A major (and wrong) conclusion of the paper is that the PAXgene method results in putatively excessive hemolysis, or, as the Figure 4 title incorrectly states, "Erythrocyte contamination". The putative difference from the other methods is described as "significantly" high, and although no statistical analysis is reported, one could hardly quibble with a ten-order-of-magnitude difference. However, this difference has nothing to do with hemolysis as a processing variable and in fact reflects well, not poorly, on the PAXgene method. The PAXgene tube is designed to lyse blood cells and stabilize RNA. This study is thus not an examination of methods, hemolysis or RBC contamination! It is simply a confirmation that lysed RBCs contain RBC RNA, whereas PBMCs do not contain RBC RNA.

2. There appears to be a fundamental confusion about what constitutes hemolysis vs. red blood cell contamination. Where is the evidence that "haemolysis has been reported to alter miRNA measurements in whole blood, plasma, serum, and tissues"? The two cited references examine the effects of hemolysis in plasma and serum, where lysis of cells can indeed have a great effect. It is clear that the very low abundance extracellular RNA fraction of blood is influenced by an influx of cellular RNA. It is unclear how degree of hemolysis could affect detection of very high abundance cellular miRNAs in whole blood (where erythrocytes dominate anyway) or in cells like PBMC that are purified away from other components.

3. The title does not describe the study. The study is presented incorrectly as a comparison of RNA extraction methods and of RNA expression in whole blood and PBMCs. Unfortunately, too many variables are changed at once in the study to allow any conclusions to be drawn. For example (see Figure 1): substrate type (whole blood vs PBMC); initial treatment (PAXgene tube for whole blood, heparin for PBMC); cell isolation (none for whole blood, centrifugation over a gradient for PBMC); and RNA isolation method (PAXgene for whole blood, two nearly identical Ambion kits for PBMC). The only variable that can be isolated is the use of the mirVana PARIS vs miRNA kit.

4. Heparin, as a PCR inhibitor, may not be the best anticoagulant for use in qPCR-based studies. No special steps are described for removal of heparin.

Minor points

1. There are no error bars to indicate biological or technical variability in Figure 3 or 4.

2. The workflow of Figure 1 does not indicate that the right-hand-side pathway involved only PBMC, not whole blood.

3. The PAXgene Blood RNA Tube and the PAXgene Blood miRNA Kit are presented as the "gold standard" in the field, yet the citation is a publication by the company, and it does not mention the miRNA kit. This should be replaced by a more objective assessment.

4. More clarity is needed in the use of terms. For example, there may or may not be RNA "expression" differences between genders or cell types. In contrast, differences in RNA quantitation observed upon freezing, or addition of PCR inhibitors, or after purification of 
the same sample with different kits, are not due to "expression" differences. The underlying expression in the cell remains the same; the ability to characterize it changes.

5. Citations of the literature are somewhat thin.

Competing Interests: No competing interests were disclosed.

I confirm that I have read this submission and believe that I have an appropriate level of expertise to state that I do not consider it to be of an acceptable scientific standard, for reasons outlined above.

Author Response 01 Dec 2014

Sadaf Atarod, Newcastle University, Newcastle upon Tyne, UK

Thanks for the valuable comments. We admit full heartedly that some descriptions and statements in the text were not as clear as they should be. This has caused misunderstanding and confusion. Good efforts have been given to improve the clarity of the text and to address each comment by the reviewer. We hope the revised manuscript could convince the reviewer (and the readers) that the design of the study is sound and rational and the conclusions can be well justified by the results.

As suggested, the results of gender and cryopreservation experiments have been excluded to deliver a more focused message.

The title of the manuscript has also been changed to clarify the message of the

1. "I would encourage the authors to remove immediately the baseless claims about putative problems with the PAXgene reagents or protocol. A major (and wrong) conclusion of the paper is that the PAXgene method results in putatively excessive hemolysis, or, as the Figure 4 title incorrectly states, "Erythrocyte contamination". The putative difference from the other methods is described as "significantly" high, and although no statistical analysis is reported, one could hardly quibble with a ten-order-of-magnitude difference. However, this difference has nothing to do with hemolysis as a processing variable and in fact reflects well, not poorly, on the PAXgene method. The PAXgene tube is designed to lyse blood cells and stabilize RNA. This study is thus not an examination of methods, hemolysis or RBC contamination! It is simply a confirmation that lysed RBCs contain RBC RNA, whereas PBMCs do not contain RBC RNA."

This article has no intention at all to claim any problems with the PAXgene protocol or to examine haemolysis or RBC contamination. The key points we intended to raise in this manuscript are 1) the use of total RNA extracted from whole blood or isolated PBMC could lead to differential results of miRNA quantification as miRNAs expressed in erythrocytes are present in total RNAs extracted from whole blood but not isolated PBMCs; 2) This inevitable fact is partly accountable for many conflicting reports in the existing literatures; 3) This is an important factor that researchers should consider when conducting miRNA research so as to extract total RNAs from most appropriate 
starting material (whole blood or isolated PBMC). Particularly, total RNAs extracted from the same starting material should be used for analysis of specific miRNAs and their downstream protein targets. These points have been clarified in the revised manuscript.

Furthermore, Figure 4 was intended to demonstrate the fact that erythrocyte related miRNA is present and detectable when using total RNAs extracted from whole blood (not to show red blood cell contamination). The title of Figure 4 and the related contents in the text have been revised to clarify this point that it is not a contamination per se but a factor that needs to be considered when analysing, reporting results and investigating miRNA protein targets.

2. "There appears to be a fundamental confusion about what constitutes hemolysis vs. red blood cell contamination. Where is the evidence that "haemolysis has been reported to alter miRNA measurements in whole blood, plasma, serum, and tissues"? The two cited references examine the effects of hemolysis in plasma and serum, where lysis of cells can indeed have a great effect. It is clear that the very low abundance extracellular RNA fraction of blood is influenced by an influx of cellular RNA. It is unclear how degree of hemolysis could affect detection of very high abundance cellular miRNAs in whole blood (where erythrocytes dominate anyway) or in cells like PBMC that are purified away from other components."

We believe this is a confusion caused by the inappropriate use of terminology rather than a flaw in study design. The revised manuscript has clarified these points. The discussion point of the manuscript is neither about haemolysis nor red blood cell contamination but simply about the fact that the presence of red blood cells in whole blood method may give rise to different miRNA quantification results compared to those where isolated PBMCs were used to extract total RNAs.

3. "The title does not describe the study. The study is presented incorrectly as a comparison of RNA extraction methods and of RNA expression in whole blood and PBMCs. Unfortunately, too many variables are changed at once in the study to allow any conclusions to be drawn. For example (see Figure 1): substrate type (whole blood vs PBMC); initial treatment (PAXgene tube for whole blood, heparin for PBMC); cell isolation (none for whole blood, centrifugation over a gradient for PBMC); and RNA isolation method (PAXgene for whole blood, two nearly identical Ambion kits for PBMC). The only variable that can be isolated is the use of the mirVana PARIS vs miRNA kit."

This study was not a comparison of RNA extraction methods. The aim of this study was to find out whether miR-146a-5p and miR-155-5p expression detected in PBMCs mirrored that quantified in total RNA extracted from whole blood. For this reason two main extraction methods had to be used one for extraction from whole blood and the other from PBMCs. Our results clarified the misconceptions reported in the literature suggesting that the levels of miRNAs detected in whole blood and PBMCs correlate with each other. In fact miR-146a-5p and miR-155-5p are both miRNAs that are detectable in acellular sources such as serum and plasma which again reiterates that the miRNA expression quantified in whole blood would be varied from that of PBMCs. 
4. "Heparin, as a PCR inhibitor, may not be the best anticoagulant for use in qPCR-based studies. No special steps are described for removal of heparin."

The use of Heparin in this study was driven by the logic that Heparin is one of the most commonly used anti-coagulants for collecting PBMCs from clinical samples. We reported over $90 \%$ efficiency of the PCR reactions demonstrating the fact that the data presented in this study had not been compromised by the use of Heparin. The quality of RNA was also high as the $260 / 280$ ratios were above 1.8 as stated in the manuscript.

Minor points

1. "There are no error bars to indicate biological or technical variability in Figure 3 or 4. "

Error bars have been added to illustrate technical variability in Figure 3 and Figure 4, respectively. As the variability is very small the error bars are hardly visible, hence inserted the sentence "The error bars represent technical variability" in the figure legends.

2. "The workflow of Figure 1 does not indicate that the right-hand-side pathway involved only PBMC, not whole blood."

The workflow of Figure 1 has been modified with an added step to indicate that the right-hand-side pathway involved only PBMCs and not whole blood.

3. "The PAXgene Blood RNA Tube and the PAXgene Blood miRNA Kit are presented as the "gold standard" in the field, yet the citation is a publication by the company, and it does not mention the miRNA kit. This should be replaced by a more objective assessment."

The company reference has been substituted with an original research article by Viprey et al (2012) which highlights the reliability and suitability of the whole blood collection method using the PAXgene Blood RNA tubes. It is worth noting that the focus of this report is to evaluate the effect of using total RNAs collected via the PAXgene Blood RNA protocol on miRNA quantification rather than on mRNA expressions.

4. "More clarity is needed in the use of terms. For example, there may or may not be RNA "expression" differences between genders or cell types. In contrast, differences in RNA quantitation observed upon freezing, or addition of PCR inhibitors, or after purification of the same sample with different kits, are not due to "expression" differences. The underlying expression in the cell remains the same; the ability to characterize it changes."

Great attention has been given to using terms with clarity and accuracy in the revised manuscript.

5. "Citations of the literature are somewhat thin."

In contrast to mRNA research where large pools of literatures have investigated 
numerous factors that could impact on the outcome of mRNA quantification, the miRNA research is still at its infancy and very few studies have investigated the impact of various total RNA extraction methods on the outcome of miRNA quantification although many articles have been published reporting associations of differential miRNA expression with diagnostic or prognostic status of diseases. This manuscript is particularly aimed to inform the miRNA community that different sources and methods used for extracting total RNAs could have significant impact on the outcome of miRNA quantification.

Competing Interests: No competing interests to declare.

The benefits of publishing with F1000Research:

- Your article is published within days, with no editorial bias

- You can publish traditional articles, null/negative results, case reports, data notes and more

- The peer review process is transparent and collaborative

- Your article is indexed in PubMed after passing peer review

- Dedicated customer support at every stage

For pre-submission enquiries, contact research@f1000.com 